\title{
Diseño de JIGS para el Area de Pintura mediante Solidworks®
}

\section{Design JIGS for the Paint Area using Solidworks®}

\author{
HERNANDEZ-BRIONES, Alejandro*† \& DIAZ-ROBLES, Raymundo
}

Universidad Tecnológica de Coahuila. Av. Industria Metalúrgica No. 2001, Parque Industrial Ramos Arizpe. C.P. 25000

ID $1^{\text {er }}$ Autor: Alejandro, Hernandez-Briones

ID $1^{\text {er }}$ Coautor: Raymundo, Diaz-Robles

DOI: $10.35429 /$ JCE.2019.10.3.27.31 Recibido 03 de Septiembre, 2019; Aceptado 30 Diciembre, 2019

\section{Resumen}

El dibujo es un lenguaje o medio de comunicación que permite dar a conocer ideas o mensajes mediante el uso de imágenes, figuras o símbolos; por esta razón, también se le denomina representación gráfica. Hoy en día, las computadoras han simplificado enormemente la manera en que diseñamos y fabricamos piezas o componentes mecánicos. El termino dibujo asistido por computadora (CAD, Computer Aided Design) se refiere a un conjunto de tecnologías basadas en el uso de computadoras para crear, analizar, y optimizar el diseño en el campo de la ingeniería. Los programas CAD proporcionan una interfase grafica de usuario (GUI, Graphics User Interface) que le permite al usuario introducir y manipular objetos geométricos en 2 y 3 dimensiones, crear dibujos de ingeniería, hacer análisis básico como el cálculo de las propiedades de masa, así como visualizar y manipular componentes individuales o ensamblajes que representan mecanismos o maquinaria cada vez más compleja. El desarrollo de los sistemas CAD en los últimos 50 años ha ido paralelo al de la tecnología de las computadoras (Jensen, 2004), y encontramos diversas aplicaciones en todos los campos de la ciencia, siendo la industria automotriz y la aeroespacial sus principales propulsores. El presente trabajo trata del uso de Solidworks ${ }^{\circledR}$ para la elaboración de JIGS (armazones) para diferentes componentes plásticos automotrices que habrán de pasar al área de pintura y posteriormente a un proceso de horneado, sin que estas se deformen.

'CAD', 'CAM', 'JIGS', Lamina de ingeniería

\begin{abstract}
Drawing is a language or half of Communication that allows you to make known ideas or messages by using images, figures or symbols; for this reason, it is also called graphic representation. Today, computers have greatly simplified the way we design and manufacture parts or mechanical components. The term Computer Aided design (CAD) refers to a set of technologies based on the use of computers to create, analyse, and optimize the design in the field of engineering. CAD programs provide a graphical user interface (GUI) that allows the user to enter and manipulate geometric objects in 2 and 3 dimensions, create engineering drawings, do basic analysis as the calculation of Mass properties, as well as visualizing and manipulating individual components or assemblies that represent mechanisms or machinery that are increasingly complex. The development of CAD systems in the last 50 years has been parallel to computer technology (Jensen, 2004), and we find various applications in all fields of science, the automotive industry and aerospace its main Propellers. The present work It deals with the use of Solidworks ${ }^{\circledR}$ for the elaboration of JIGS (frames) for different automotive plastic components that will have to move to the painting area and then to a baking process, without these deforming.
\end{abstract}

CAD, CAM, JIGS, Engineering sheet

Citación: HERNANDEZ-BRIONES, Alejandro \& DIAZ-ROBLES, Raymundo. Diseño de JIGS para el Area de Pintura mediante Solidworks®. Revista de Ingeniería Civil. 2019. 3-10: 27-31

\footnotetext{
* Correspondencia del Autor (correo electrónico: ahbriones@utc.edu.mx)

$\dagger$ Investigador contribuyendo como primer autor.
} 


\section{Introducción}

El diseño asistido por computadora (CAD, computer aided design) se refiere al uso de las computadoras, mediante un software o programa específico, para crear la geometría (el diseño) de una pieza especifica. A su vez, la manufactura asistida por computadora (CAM, computer aided manufacturing) es el uso de las computadoras para definir las trayectorias de las herramientas que permiten crear una pieza mediante diferentes procesos de fabricación. Así, las técnicas del CAD-CAM permiten a los fabricantes administrar y controlar todos los aspectos relacionados con el proceso de fabricación de una pieza o componente mecánico: el diseño, prueba, validación y fabricación de la pieza. Mediante el uso de técnicas de CAD-CAM se consigue abaratar costos, aumentar la calidad y reducir el tiempo de diseño y producción. Estos tres factores son vitales para la industria actual.

\section{Justificación}

La industria de la región se conforma por empresas relacionadas con el sector automotriz donde constantemente se requieren herramientas de innovación y mejora en el diseño de productos. En la región sureste del estado de Coahuila el clúster automotriz tiene gran relevancia, ya que se cuenta con más de 3,000 empresas en diferentes rubros. Existen diferentes compañías, tanto pequeñas y medianas empresas, las cuales dan servicio a empresas grandes que soportan el crecimiento de la región (SEDECT, 2017).

\section{Problema}

El diseño y la fabricación asistidos por computadora han alcanzado actualmente un gran nivel de desarrollo e implantación. El presente trabajo se centra en el diseño de unos armazones denominados JIGS para una empresa de la región, y que actualmente es proveedora de la industria automotriz. El proceso de investigación considera los parámetros solicitados por el cliente, la mejor alternativa para la manipulación de la pieza, evaluación del material y un diseño sencillo para su posterior fabricación

\section{Hipótesis}

Existe la necesidad de generar y sustentar alternativas de solución a los problemas que se tengan en la industria relacionados con el diseño de piezas y productos, así como el desarrollo de dispositivos y mecanismos para su operación. Se plantea que si el personal de la industria, las pequeñas y medianas empresas, los talleres que proveen de alguna forma a las empresas de la región, así como los ingenieros recién egresados de las diversas carreras de ingeniería conocieran, dominaran y aplicaran las técnicas del CAD$\mathrm{CAM}$, los trabajos se harían más rápido y con mayor precisión; lo que contribuiría a la mejora en el desarrollo de solución de problemas, que es lo que se pretende.

\section{Objetivos}

\section{Objetivo General}

Plantear un procedimiento para el análisis de partes y componentes automotrices, para posteriormente generar una alternativa de solución en el diseño de armazones o JIGS para soporte y sujeción de piezas, así como un análisis de diseño mediante un software CAD.

\section{Objetivos específicos}

- $\quad$ Definir un procedimiento para el diseño de JIGs que facilitan los procesos de fabricación en la industria de la región.

Capacitar al personal de la industria de la región, asi como estudiantes de Ingenieria, en estas técnicas que permiten eficientar los procesos $\mathrm{y}$ mejorar la calidad y productividad de estas.

\section{Marco Teórico}

El desarrollo de los sistemas CAD industriales empezó en la década de los años sesenta en la industria automotriz y la aeroespacial. Fue hasta la década de los años noventa que se desarrollaron paquetes $\mathrm{CAD}$ más avanzados para 3D que usaban modelado sólido $\mathrm{y}$ superficies NURBS (superficie B racional no uniforme). La integración del CAD a la ingeniería y a la manufactura fue enormemente favorecida por el desarrollo de las redes de alta velocidad y de Internet (Jensen, 2004). 


\section{Acerca del programa de Solidworks}

SolidWorks es una solución de diseño tridimensional completa que integra un gran número de funciones avanzadas para facilitar el modelado de sólidos en 3D y superficies avanzadas, crear grandes ensamblajes, generar planos, así como otras funcionalidades que permiten validar, gestionar y comunicar proyectos de forma rápida, precisa y fiable (Gómez, 2016). Contiene tres módulos: Pieza, Ensamblaje y Dibujo.

\section{Metodología de Investigación}

Cuando se recibe una pieza a la que se le desea fabricar un soporte o armazón de sujeción, denominado JIG o bien Fixture, es muy importante conocer las especificaciones para su elaboración. Denominadas Specs (del término en ingles Specifications), estas determinan las limitaciones y valores de control para su elaboración. Así, sin utilizar un estudio de optimización, el mejor diseño de piezas solo puede lograrse mediante costosos y largos ciclos de desarrollo de productos (Iancu, 2017).

La investigación aplicada busca establecer los pasos para definir un procedimiento estándar que permita construir un modelo en el sistema CAD de SolidWorks, probarlo en conjunto con la pieza a ensamblar, y modificar el diseño en función de los resultados de la simulación y prueba de funcionamiento. La experimentación se lleva a cabo en forma virtual.

\section{Tipo de Investigación}

El interés principal es revisar el estado del arte en cuanto al uso de un software de diseño mecánico y su uso en la industria de la región. Para el presente ensayo se plantea el procedimiento para elaborar un armazón de sujeción, sin embargo, en la etapa experimental se recibió un total de ocho propuestas para su diseño, prueba y posterior fabricación.

\section{Métodos Teóricos}

\section{¿Qué es un JIG?}

Un JIG o armazón es una estructura firme y sólida de tal modo que sostiene a otras piezas o sirve de base para que se monte sobre ella otra cosa.
Es lo que soporta a otro objeto. Este tipo de armazones se utilizan para la sujeción o soporte de piezas en el sector automotriz, sea para una operación de pintura, recubrimiento o ensamblado (p.e. un arnés o el cableado eléctrico del panel frontal). Mediante un sistema CAD como SolidWorks, se pueden realizar tareas como probar el modelo utilizando la computadora en lugar de pruebas de campo, lo que reduce el costo y reduce el tiempo de comercialización reduciendo el número de ciclos de desarrollo de productos y optimizar los diseños mediante la simulación de conceptos y escenarios antes de tomar decisiones finales (Ianku, 2017).

\section{Desarrollo del Procedimiento}

El Procedimiento planteado se conforma de las siguientes etapas:

1. Evaluación de la pieza o parte recibida. Se evalúa la geometría: puntos de reforzamiento, así como de sujeción o clípeo (para el agarre o posicionamiento de la pieza). Esto permite la definición de operaciones a utilizar en el software para la elaboración del armazón.

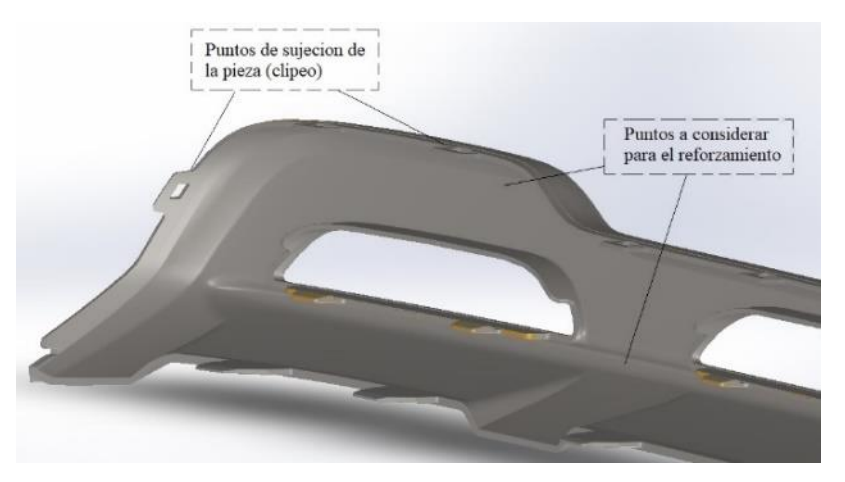

Figura 1 Análisis de geometría para generación de alternativas

2. Generación de geometría 3D en base a la forma proporcionada. Se procede a la elaboración del armazón, considerando la forma geométrica de la pieza. Al realizar el ensamblado, se evalúan los puntos a reforzar, los puntos de sujeción y retiro de esta sin llegar a dañar la pieza original. El programa permite trabajar curvas en $3 \mathrm{D}$, denominadas spline $\mathrm{o}$ forma libre; esto facilita el trazado encima de la pieza proporcionada y ajustarlo a dicha geometría. 


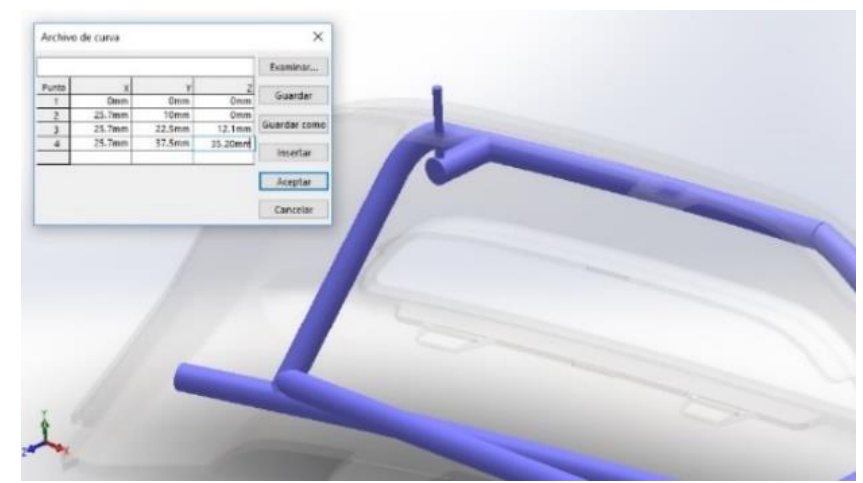

Figura 2 Trazado de spline para generar armazón

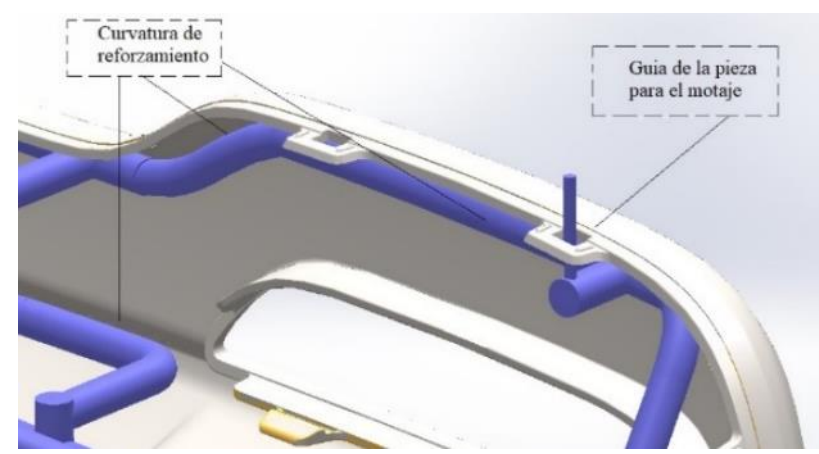

Figura 3 Evaluación de las áreas de apoyo

3. Revisión de especificaciones (Specs). En este paso, es importante definir los parámetros de trabajo, tales como área de pintura que cubre el robot, distancia entre piezas, posicionamiento en el armazón y numero de ensambles, tanto en el eje $X$ como en el eje Y, así como el ángulo de posicionamiento a aplicar. Esto se realiza al probar un arreglo de JIGs, determinando el posicionamiento en el espacio 3D.

4. Validación y liberación del diseño. El software CAD permite determinar el peso de la pieza, de acuerdo con la densidad del material especificado (Figura 4). A su vez, permite la evaluación del modelo mediante un Estudio de Diseño. La interfaz del asistente guía a través de un proceso paso a paso para especificar variables, restricciones y objetivos, ejecutar el estudio y ver los resultados, pudiendo evaluar el Factor de seguridad, el desplazamiento máximo y el estrés máximo (Figura 5).

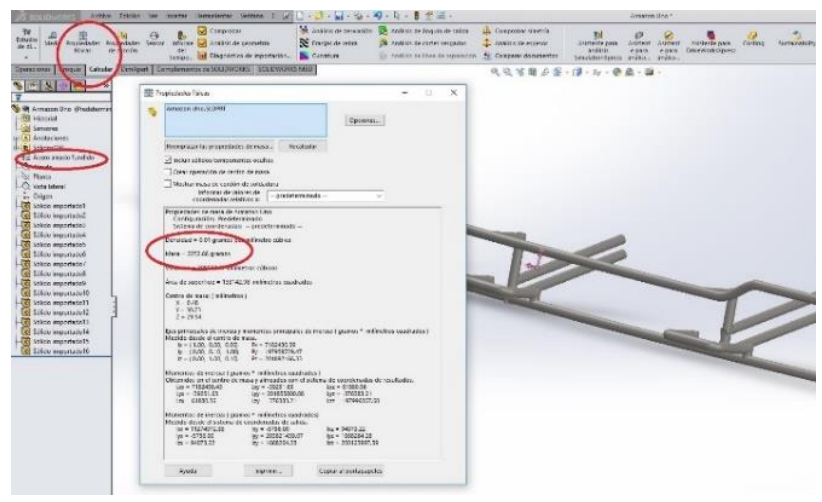

Figura 4 Calculo de masa

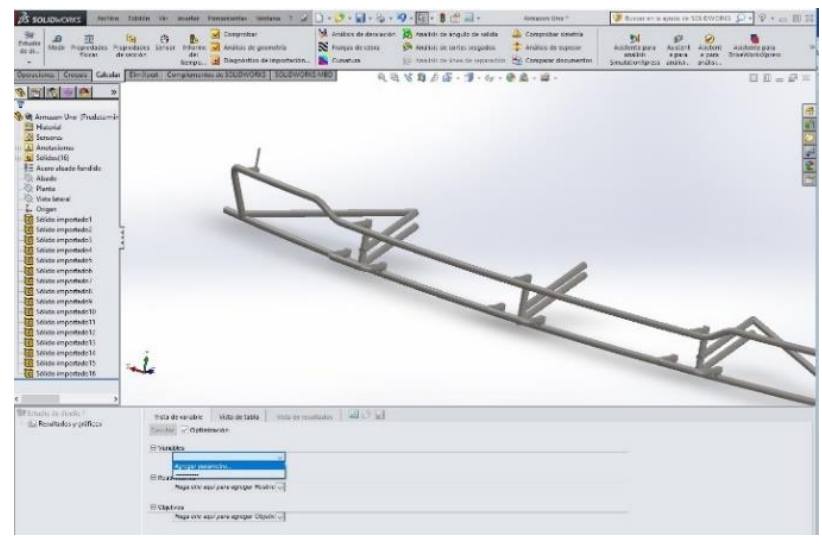

Figura 5 Asistente para Estudio de Diseño

A continuación, se muestra un estudio para hacer una optimización dimensional, usando como objetivo la minimización de la masa. Los pasos son:

a) En la ventana del asistente, seleccionar Agregar variables...

b) Dar clic en una parte de la geometría, y posteriormente, seleccionar su dimensión (acotación). Puede agregar varios valores para el estudio.

c) Enseguida, seleccione la opción Restricciones y el sensor de Masa (este sugiere el peso inicial. Presione Aceptar.

d) Por último, seleccione la Meta propuesta: Maximizar o Minimizar el peso de la pieza

Al correr la simulación, el software habrá de generar diferentes escenarios, el cambio de dimensiones en la pieza es proporcional al peso de la masa inicial. 


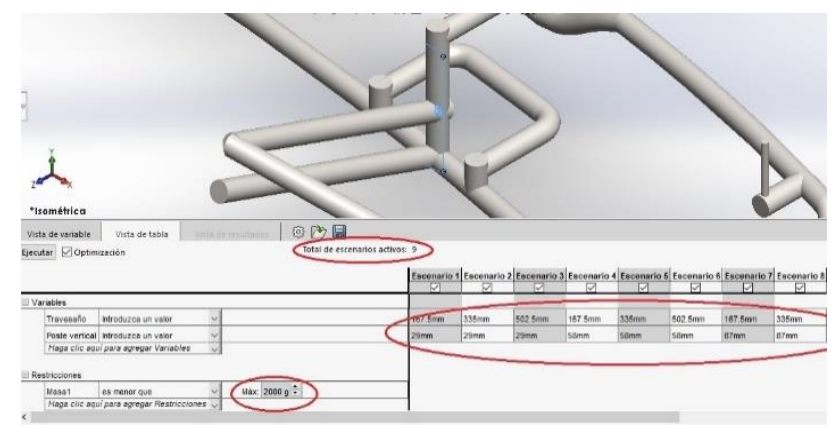

Figura 6 Resultados de la corrida con 9 escenarios posibles

Para dicho estudio, Solidworks sugiere nueve posibles combinaciones para alcanzar el objetivo propuesto: Disminuir de 2252.66 gramos a 2000 gramos $(2 \mathrm{~kg})$, con diferentes modificaciones a dimensiones de la pieza sin afectar su funcionalidad y seleccionando la mejor combinación para mantener los puntos clave del diseño.

5. Envío a fabricación de componentes y ensamblado. Se definen los procesos de fabricación, sugerencia de materiales y espesores (comúnmente varilla de acero con $3 / 8$ " de espesor). Para el presente JIG, se lleva a cabo un proceso de conformado, posteriormente unión por soldadura de arco eléctrico.

\section{Resultados}

Además de la funcionalidad básica de creación de piezas, ensamblajes y dibujos, SolidWorks permite realizar otro tipo de operaciones variadas que facilitan el proceso de diseño, simulación y gestión de proyectos. Bajo el nombre de complementos se presentan algunas funcionalidades o herramientas que facilitan el proceso de diseño y a la vez disminuyen el elevado costo del diseño de componentes y la disminución del tiempo invertido.

\section{Referencias}

Gómez González Sergio. (2016). Solidworks Practico I. Ed. Alfaomega, Ediciones técnicas Marcombo.

Gómez González Sergio. (2017). Solidworks Practico II. Ed. Alfaomega, Ediciones técnicas Marcombo.

Iancu, C. (2017). About optimization Design Study on SolidWorks, Fiability \& Durability Revue. ISSN 1844-640X, 1/2017
Jensen Cecil \& Helsel Jay D. (2004). Dibujo y diseño en Ingenieria. Ed. McGrawHill.

Lombard, M. (2013). SolidWorks Bible, Wiley $\&$ Sons, USA.

SolidWorks Manual del Usuario (2017). Dassault Systèmes SolidWorks Corporation, Waltham, MA, USA.

Tickoo Sham (2018). Solidworks 2018 for Designers. Ed. CADCIM Technologies, USA. 